Review

\title{
Leaf Primordia-Free Shoot Apical Meristem Culture: A New Method for Production of Viroid-Free Plants
}

\author{
Munetaka Hosokawa \\ Graduate School of Agriculture, Kyoto University, Sakyo-ku, Kyoto 606-8502, Japan
}

\begin{abstract}
Viroids are the smallest and one of the most problematic pathogens known in horticulture. Despite not coding for proteins they replicate in nuclei or chloroplasts using host enzymes. From many pathological studies, it is obvious that viroids are completely different agents from viruses. For the past 40 years, elimination of viroids from infected plants has been the main theme in horticulture and several approaches have been established; however, these procedures remain complicated. Leaf primordia-free shoot apical meristem culture is a newly established method that can effectively eliminate viroids. Therefore, in this review, the method is summarized and the mechanism of viroid elimination is discussed.
\end{abstract}

Key Words: Avsunviroidae, Pospiviroidae, shoot apical meristem, tissue culture, viroid.

\section{Introduction}

Viroids are the smallest known pathogens, consisting of 246-401 nucleotides, and are responsible for infectious diseases in various crops. These pathogens are circular, single-stranded RNAs without any apparent coding capacity and completely depend on host enzymes for their replication (Diener, 1991). To date, about 30 different viroids, classified into two families, Pospiviroidae and Avsunviroidae, according to their features, have been reported (Flores et al., 2000b). Viroids replicate through rolling circle transcription, which results in minus-strand multimeric RNA synthesis. Pospiviroidae possesses a thermostable rod-like structure with a central conserved region and cannot selfcleave (Steger and Riesner, 2003). Viroids that belong to Pospiviroidae proliferate and accumulate in the nucleus (Semanic et al., 1976) using DNA-dependent RNA polymerase II. Viroids belonging to Avsunviroidae proliferate and accumulate in chloroplasts using NEP polymerase coded by the nuclear gene. They can cleave the multimeric product that is transcribed by rolling circle transcription to unit lengths from their ribozyme activity (Flores et al., 2000a). Now, there are three streams in viroid research: viroid pathology, e.g., by Flores et al. (2005) or Tabler and Tsagris (2004), RNA traffic research (Ding and Itaya, 2007; Ding et al., 2005),

Received; July 15, 2008. Accepted; August 8, 2008.

E-mail: mune@kais.kyoto-u.ac.jp. and cleaning or protection horticultural crops from viroids (Barba et al., 2003b). Although the first two research areas are of interest, they are not dealt with in this review.

Viroid-infected plants are spread not only through vegetative propagules, but also through crude sap on knives and pruning tools, seeds or pollen, and sometimes by insects. When Potato spindle tuber viroid (PSTVd) is co-infected with velvet tobacco mottle virus, PSTVd is encapsulated by the virus and transmitted by mirid (Francki et al., 1986). Moreover, PSTVd is transmitted by Myzus persicae when it is co-infected with potato leafroll virus (Salazar et al., 1995). Even if there is no economic loss due to symptomless infection, problems arise when the viroid is transmitted to other plant species, and clear symptoms occur. In addition, if only $1 \%$ viroidinfected plants mix with healthy propagules, a huge cost is involved in separating out infected plants (Randles, 2003). Thus, the complete eradication of problematic viroids, including the detection of unknown viroid species, is an important criterion for horticulture research.

For viroid elimination, several strategies are reported (Barba et al., 2003b). High or low temperature treatments are often effective for eliminating viroids, with some exceptions; however, temperature treatments are usually needed for long duration e.g., Apple scar skin viroid was eliminated from apples after 70 days of treatment at $38^{\circ} \mathrm{C}$ (Howell et al., 1998). For low temperature treatment, $2-4^{\circ} \mathrm{C}$ treatment for 8 months produced $50 \%$ 
Hop stunt viroid (HSVd)-free plants (Adams et al., 1996). Effects of low temperature include a decreased viroid titer of infected plants under natural conditions e.g., in chrysanthemum, some winter suckers of Chrysanthemum stunt viroid (CSVd)-infected cultivars become viroid free, caused by low winter temperatures in Japan (Sugiura and Hanada, 1998). To improve viroidfree plant production, these temperature therapies are used in combination with shoot tip culture or shoot tip grafting (Barba et al., 2003b); however, utilization of these temperature methods is limited because of the long treatment period and some viroids, such as PSTVdinfecting potato plants (Barba et al., 2003b), remain unaffected by high temperature. Another effective method for viroid elimination is chemotherapy, for which antiviral agents such as amantadine, ribavirine, or 2thiouracil are applied to the culture media for shoot tip culture or shoot tip grafting. For example, CSVd was successfully eliminated from a chrysanthemum cultivar by adding amantadine to the culture medium (Horst and Cohen, 1980).

Currently, numerous modified detection methods have been established, such as PCR hybridization, RT-PCR, nested PCR, dot blot hybridization, and loop-mediated isothermal amplification (Notomi et al., 2000), which can detect viroid infection with high sensitivity (Hirata et al., 2002). Plants which have been reported using temperature treatments alone, chemotherapy and combination methods with shoot tip culture, should be tested using these sensitive methods to ensure that they are viroid-free.
As reported for viruses, viroid-free production is also thought to be improved by dissecting small shoot tips (Barba et al., 1995; Momma and Takahashi, 1983), demonstrating the necessity for shoot regeneration from ultrasmall SAMs for complete viroid elimination from infected plants. Ball (1948) and Sussex (1952) showed the regeneration of plants from a small portion of an apical meristem that had been divided by razor blades; however, the viability of the excised portion of the meristem was not examined. Leaf primordia-free shoot apical meristems (LP-free SAMs) rapidly dry up, making culture of LP-free SAMs for plant regeneration difficult without plant growth regulators (PGRs) (Murashige et al., 1972). PGRs typically help plant regeneration in meristem culture, but sometimes growth regulators increase the occurrence of mutations, which limits their use in horticulture. Many trials resulted in a new method, LP-free SAM culture (Hosokawa et al., 2004b), by which plants can regenerate without PGRs. Therefore, in this review, the method is summarized and the mechanism of viroid elimination is discussed.

\section{Establishment of LP-free SAM culture}

A new apparatus was designed and manufactured by us for dissection of LP-free SAMs or a small portion of it (Fig. 1a). This apparatus is standardized for microsurgery, thus making it possible to dissect more uniform-sized LP-free SAMs for operators with limited skills. A shoot with all leaf primordia removed and the meristem exposed was placed on the apparatus (Fig. 1a). The cut direction of the razor blade was electrically
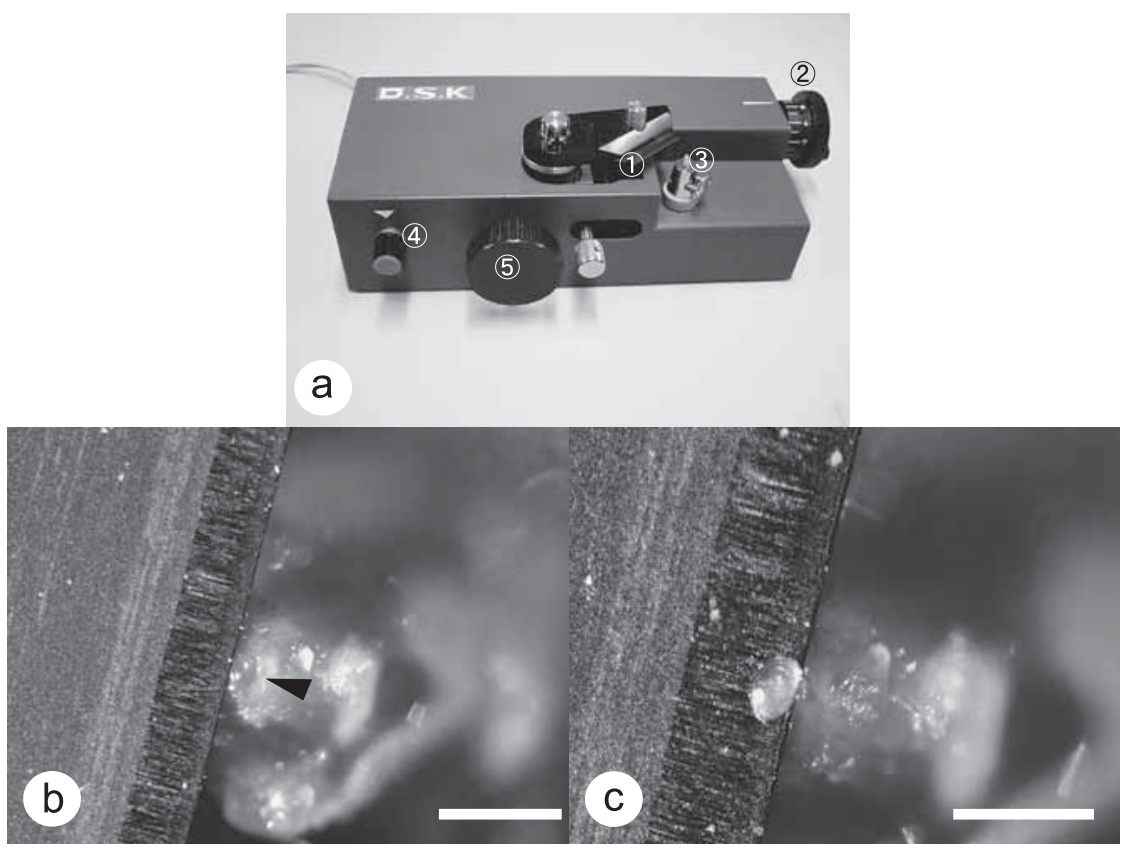

Fig. 1. Apparatus for excising LP-free SAM. a: whole of the apparatus. (1) a razor blade, which is controlled up and down by the micrometer level, (2) dial for controlling the position of the razor blade, (3) sample holder for a shoot where all leaf primordia were dissected, (4) dial for rotating the shoot to an appropriate position for excising the dome, (5) lever for moving the razor blade slowly forward to the meristem dome. b: LP-free SAM observed under a stereomicroscope, and LP-free SAM (arrowhead) that touched the razor blade is seen. c: LP-free SAM of chrysanthemum 'Iwa-no-hakusen' on a razor blade. Bar (b, c): $0.5 \mathrm{~mm}$ (Citation from Hosokawa et al., 2004b). 
Table 1. Shoot regeneration from LP-free SAMs of 'Piato' and 'Stettsuman' attached to root tips of various chrysanthemum cultivars.

\begin{tabular}{|c|c|c|c|c|c|c|}
\hline $\begin{array}{c}\text { Cultivar of LP-free } \\
\text { SAMs }\end{array}$ & Cultivar of root tips & $\begin{array}{l}\text { No. LP-free SAMs } \\
\text { attached }\end{array}$ & $\begin{array}{c}\text { No. viable exp } \\
(\%)\end{array}$ & $\operatorname{lants}^{\mathrm{y}}$ & \multicolumn{2}{|c|}{$\begin{array}{c}\text { No. shoots with }>3 \mathrm{~mm} \text { leaves }{ }^{\mathrm{x}} \\
(\%)\end{array}$} \\
\hline Piato & No root tip ${ }^{z}$ & 29 & $0(0)$ & $\mathrm{c}$ & $0(0)$ & $\mathrm{c}$ \\
\hline Piato & Sei Alps & 39 & $16(41.0)$ & $\mathrm{a}$ & $1(2.6)$ & $\mathrm{b}$ \\
\hline Piato & Mistletoe & 36 & $17(47.2)$ & $\mathrm{a}$ & $6(16.7)$ & $\mathrm{a}$ \\
\hline Stettsuman & Sei alps & 409 & $73(17.8)$ & $\mathrm{b}$ & $6(1.5)$ & $\mathrm{b}$ \\
\hline
\end{tabular}

${ }^{z}$ LP-free SAMs were directly incubated on modified Knop medium.

y Data were collected 10 days after attachment to root tips.

x Data were collected 30 days after attachment to root tips.

Values followed by the same letter in each column are not significantly different $(Z$ test: $P=0.05)$.

Citation from Hosokawa et al. (2004b).

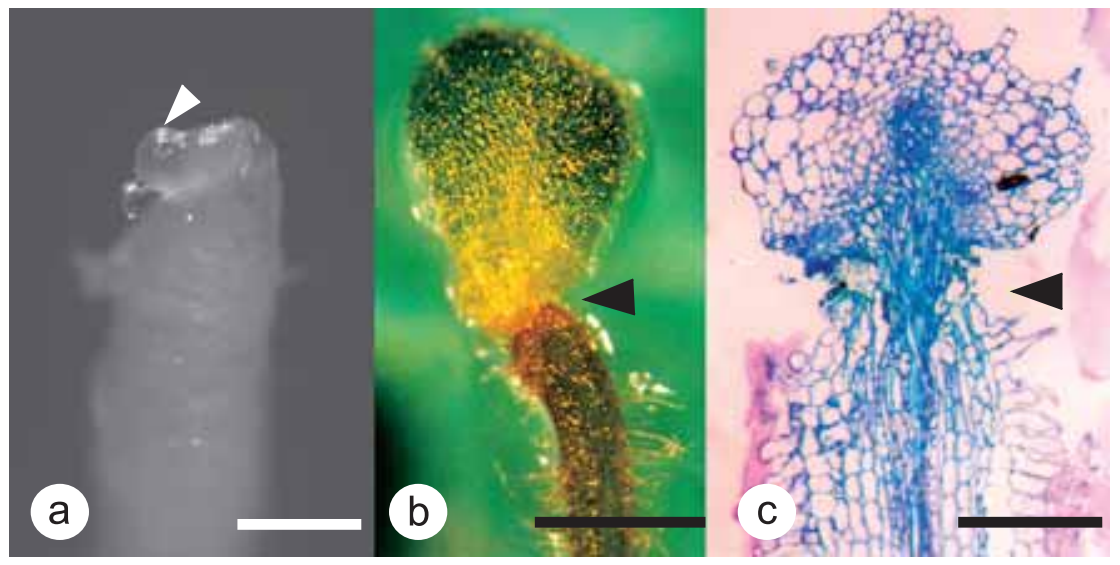

Fig. 2. Shoot regeneration from an LP-free SAM of 'Piato' chrysanthemum attached to chrysanthemum root tip 'Mistletoe'. a: The first leaf primordium on an LP-free SAM of 'Piato' attached to a root tip of 'Mistletoe' 7 days after attachment to the root tip. Arrowhead indicates the leaf primordium. b: A shoot of chrysanthemum 'Majore' with $3 \mathrm{~mm}$ leaves 32 days after attachment to a chrysanthemum root tip of 'Mistletoe'. Arrowhead indicates the attached region. c: Histological observation of the attached resion of the shoot of 'Piato' with $3 \mathrm{~mm}$ leaves grown on a root of 'Mistletoe' 30 days after attachment. Arrowhead indicates the attached region. Bar (a, c): $1 \mathrm{~mm} ; \mathrm{bar}(\mathrm{b}): 3 \mathrm{~mm}$ (Citation from Hosokawa et al. 2004b).

controlled under a stereomicroscope (Fig. 1b), and a uniform-sized LP-free SAM was excised (Fig. 1c). The excised LP-free SAM was picked up from the razor blade using a sharpened glass tube and transferred to the cut root tip surface. High humidity of LP-free SAM was maintained throughout the microsurgery using a small machine for generating ultrasonic mist $(7 \times 10 \times$ $20 \mathrm{~cm}$, Dosaka EM, Japan). Shoot regeneration was not observed from LP-free SAMs of 'Piato' that were cultured directly on modified Knop medium without PGRs and not attached to root tips (Table 1). In comparison, one week after attachment to the root tip in vitro, the first leaf primordium formed at the center of the LP-free SAM (Fig. 2a). Some explants had leaves that were over $3 \mathrm{~mm}$ in length after 30 days (Table 1 and Fig. 2b). The number of 'Piato' plants that developed $3 \mathrm{~mm}$ leaves was higher when 'Piato' LP-free SAMs were attached to 'Mistletoe' root tips as compared to those of 'Sei Alps'. When LP-free SAMs of cultivar 'Stettsuman' were attached to root tips of 'Sei Alps', only $1.5 \%$ of the LP-free SAMs developed viable shoots with $3 \mathrm{~mm}$ leaves after 30 days of incubation (Table 1). Complete vascular connection was observed when a

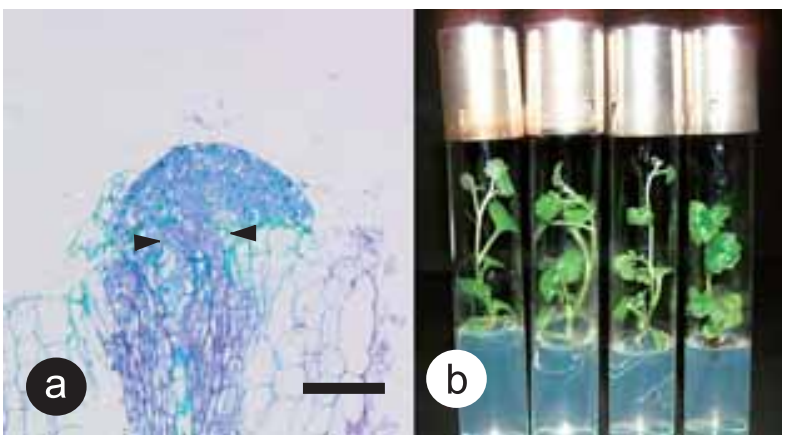

Fig. 3. Shoot regeneration from an LP-free SAM of chrysanthemum attached to a cabbage root tip. a: Histological observation of an attached region between the chrysanthemum 'Piato' LP-free SAM and cabbage root tip 7 days after attachment. Bar: $0.1 \mathrm{~mm}$. b: Plants 'Piato' developed on modified MS medium 50 days after subculture (Citation from Hosokawa et al., 2004b).

'Piato' shoot regenerated on a 'Mistletoe' root tip (Fig. 2c). When the root tips of 'Sei Alps' were used instead of 'Mistletoe' with 'Piato' LP-free SAMs, almost all explants were removed easily from the root tips after 10-day incubation, indicating the lack of a vascular connection (data not shown). It is important to select an 
appropriate chrysanthemum cultivar for the root tips; however, many viroids are reported to be seed-borne (Mink, 1993), e.g., CSVd in chrysanthemum (Ohishi et al., 2001). Since the screening of CSVd-free chrysanthemum cultivars which can be used for root tips is difficult, other plant species for root tips should be screened.

LP-free SAMs of 'Piato' chrysanthemum were attached to root tips obtained from in vitro seedlings of cabbage (Brassica oleracea), petunia (Petunia $\times$ hybrida), and carnation (Dianthus caryophyllus), 2$3 \mathrm{~mm}$ in length. Among these plant species, cabbage root tips were the most suitable for small plantlet regeneration from chrysanthemum LP-free SAM (Table 2), and the survival rate of LP-free SAMs attached to cabbage root tips was similar to that of chrysanthemum root tips (Tables 1 and 2). Root tips of petunia and carnation were not suitable for the survival of LP-free SAMs of chrysanthemum. Chrysanthemum LP-free SAMs and cabbage root tips partially adhered 10 and 30 days after attachment, and microscopic observation showed that the vasculature of the two organs was not connected (data not shown). Shoots on cabbage roots did not have expanded leaves greater than $3 \mathrm{~mm}$ long after 30-day incubation (Table 2); hence, these small chrysanthemum shoots were removed from the cabbage roots and then subcultured on modified MS medium without PGRs. Thirty days after subculture, $30 \%$ of total subcultured shoots had expanded $3 \mathrm{~mm}$ leaves (Table 2 and Fig. 3b).

Three cultivars of chrysanthemum were used to culture LP-free SAMs attached to cabbage root tips. LP-free SAMs of these cultivars were attached to root tips of 'Shikidori' cabbage sown in vitro, and maintained viability on cabbage root tips (Table 3 ). The survival rates were $63-76 \%$ among the cultivars. One month after attachment, viable chrysanthemum shoots were removed from cabbage roots and subcultured on modified MS medium without PGRs. Chrysanthemum plantlets developed normally, and $47-69 \%$ of the plants regenerated phenotypically into normal plants.

Root tips have some advantages for rescuing LP-free SAMs for the following reasons. 1) The root tip has a high rate of cell division, 2) the two organs may adhere tightly, because excessive callus is not formed on the cut surface of root tips, and 3) root tips of some plants, such as cabbage, have little ability to distinguish between themselves and another species. LP-free SAMs adhered to cabbage roots temporarily (Fig. 3a). When LP-free

Table 2. Shoot regeneration from LP-free SAMs of 'Piato' attached to root tips of various plant species.

\begin{tabular}{|c|c|c|c|c|c|}
\hline Plant for root tips & $\begin{array}{c}\text { No. LP-free } \\
\text { SAMs attached }\end{array}$ & $\begin{array}{l}\text { Rate of viable } \\
\text { meristems }(\%)^{z}\end{array}$ & $\begin{array}{l}\text { No. plants with } \\
>3 \mathrm{~mm} \text { leaves }^{\mathrm{y}}\end{array}$ & $\begin{array}{l}\text { No. plants } \\
\text { subcultured }^{\mathrm{x}}\end{array}$ & $\begin{array}{c}\text { Percentage of plants with } \\
>3 \text { mm leaves } \\
\text { after subculture }(\%)\end{array}$ \\
\hline No root tip & 15 & $0 \quad \mathrm{c}$ & 0 & 0 & - \\
\hline Cabbage & 31 & $71.0 \mathrm{a}$ & 0 & 21 & 29.0 \\
\hline Petunia & 26 & $15.4 \mathrm{~b}$ & 0 & 4 & 84.6 \\
\hline Carnation & 23 & $0 \quad \mathrm{c}$ & 0 & 0 & - \\
\hline Chrysanthemum & 33 & $69.7 \mathrm{a}$ & 8 & 23 & 30.3 \\
\hline
\end{tabular}

${ }^{z}$ Data were collected 10 days after attachment to root tips.

y Data were collected 30 days after attachment to root tips just before subculture on modified MS medium.

${ }^{x}$ No. plants transferred to modified MS medium 30 days after attachment to root tips.

Data were collected 30 days after subculture on modified MS medium.

(No. plants with $3 \mathrm{~mm}$ leaves/No. total subcultured plants) $\times 100$

Values followed by the same letter in the column are not significantly different ( $\mathrm{Z}$ test: $P=0.05)$.

Citation from Hosokawa et al. (2004b).

Table 3. Shoot regeneration of various chrysanthemum cultivars from LP-free SAMs attached to cabbage root tips.

\begin{tabular}{|c|c|c|c|c|c|c|}
\hline Shoot cultivar & Culture period & $\begin{array}{c}\text { No. LP-free SAMs } \\
\text { attached }\end{array}$ & $\begin{array}{c}\text { Viable explants }{ }^{\mathrm{z}} \\
(\%)\end{array}$ & $\begin{array}{l}\text { No. plants with }> \\
3 \mathrm{~mm} \mathrm{leaves}^{\mathrm{y}}\end{array}$ & $\begin{array}{l}\text { No. plants } \\
\text { subcultured }^{\mathrm{x}}\end{array}$ & $\begin{array}{c}\text { No. plants with }>3 \mathrm{~mm} \\
\text { leaves after } \\
\text { subculture }(\%)^{\mathrm{w}}\end{array}$ \\
\hline Piato & $2001.12-2002.4$ & 180 & $65.6 \mathrm{a}$ & 0 & 115 & $79(68.8) \mathrm{a}$ \\
\hline Iwa-no-hakusen & $2001.12-2002.3$ & 261 & $62.8 \mathrm{~b}$ & 0 & 88 & $41(46.7) \mathrm{b}$ \\
\hline Stettsuman & 2002. 1-2002.3 & 125 & $76.0 \mathrm{a}$ & 0 & 23 & $12(52.2) \mathrm{a}$ \\
\hline
\end{tabular}

${ }^{z}$ Data were collected about 10 days after incubation.

y No. plantlet with over $3 \mathrm{~mm}$ leaves

Data were collected 30 days after attachment just befor subculture on modified MS medium.

${ }^{x}$ No. plantlet transferred to modified MS medium 30 days after attachment to root tips.

${ }^{w}$ Data were collected 30 days after subculture on modified MS medium.

(No. plants with over $3 \mathrm{~mm}$ leaves/No. total subcultured plants) $\times 100$

Values followed by the same letter in the same column are not significantly different ( $\mathrm{Z}$ test: $P=0.05)$.

Citation from Hosokawa et al. (2004b). 
SAMs produced a few leaf primordia, these small shoots could be easily removed from cabbage roots, possibly because the cabbage root recognizes differentiated organs such as a chrysanthemum vascular bundle, and adhesion between the cabbage root and the chrysanthemum plant does not occur. Similar results were obtained when chrysanthemum LP-free SAMs were attached to Brassica campestris root tips (unpublished data). Because cabbage root tips are used for LP-free SAM culture of tomato (Hosokawa et al., 2004d), petunia (unpublished data), dahlia (unpublished data), root tips of cabbages have general versatility for LP-free culture.

\section{Elimination of viroids using LP-free SAM culture}

\section{CSVd as a model case for Pospiviroidae}

Two main viroids infect chrysanthemum (Dendranthema grandiflorum): CSVd (Pospiviroidae) and Chrysanthemum chlorotic mottle viroid (CChMVd) (Avsunviroidae). CSVd infection of many chrysanthemum cultivars is a severe problem worldwide (Randles, 2003). Although the magnitude of CSVd infection of chrysanthemum plants varies between cultivars, the general symptoms are as follows (Brierley and Smith, 1949; Dimock, 1947; Hollings and Stone, 1973; Horst and Langhans, 1977): young leaves turn pale green, plant height decreases (stunting), the anthocyanin in stems disappears, leaves and flowers become small, and rooting ability decreases. On the other hand, the problem of CChMVd infection in chrysanthemum cultivation is currently restricted to a small area (Flores et al., 2003); however, CChMVd infection has recently been reported in Japanese chrysanthemum cultivars (Hosokawa et al., 2005; Yamamoto and Sano, 2005) and chrysanthemum cut flowers imported from Europe (Hosokawa et al., 2005). CChMVd diseases were observed in young leaves, i.e., young leaves had turned pale green (Flores et al., 2003).

When LP-free SAM culture was applied for CSVd elimination, $28.6 \%$ of the total number of tested regenerants did not have a band corresponding to CSVd by RT-PCR (Table 4). Moreover, the nested PCR band corresponding to CSVd was not detected in $14.3 \%$ of regenerated shoots (Table 4). All plants determined to be CSVd-free by nested PCR were also confirmed to be CSVd-free by RT-PCR followed by dot blot hybridization. Plants that did not show the band following nested PCR are thought to be CSVd-free plants. In these free plants, CSVd was not detected 2 years after this first test. Among the many high sensitive detection methods for viroids, nested PCR is one of simple and low cost methods with highest sensitivity; hence, we used this method through our experiment. CSVd was detected in all the shoots regenerated from LP-containing SAMs by nested PCR (Table 4). In conclusion, to eliminate CSVd from highly infected chrysanthemum cultivars, it is important to regenerate plants from LP-free SAMs.

To improve the regeneration rate of CSVd-free plants, it was examined the effect of repeating LP-free SAM culture. 'Piato' shoots were regenerated on cabbage root tips and subcultured on modified MS medium. Of the 115 shoots subcultured on modified MS medium, 79 shoots $(68.7 \%)$ grew $3 \mathrm{~mm}$ expanded leaves (Table 5).

Table 4. CSVd-free plant production by LP-free SAM culture of 'Piato' attached to root tips of chrysanthemum.

\begin{tabular}{lcccc}
\hline \hline No. of leaf primordia & $\begin{array}{c}\text { No. of plants used for CSVd } \\
\text { detection }\end{array}$ & $\begin{array}{c}\text { No. of plants without CSVd signal } \\
\text { detected by RT-PCR }(\%)^{\mathrm{z}}\end{array}$ & $\begin{array}{c}\text { No. of CSVd-free plants detected } \\
\text { by nested PCR }(\%)^{\mathrm{y}}\end{array}$ \\
\hline LP-containing SAM $^{\mathrm{x}}$ & 14 & $2(14.3) \mathrm{b}$ & $0(0)$ & $\mathrm{b}$ \\
LP-free SAM & 21 & $6(28.6) \mathrm{a}$ & $3(14.3) \mathrm{a}$ \\
\hline
\end{tabular}

z (No. of plants without CSVd signal detected by RT-PCR/No. of plants used for CSVd detection) $\times 100$

y (No. of CSVd-free plants detected by nested PCR/No. of plants used for CSVd detection) $\times 100$

${ }^{x}$ LP-containing SAM means a SAM with one or two leaf primordia.

Values followed by the same letter in the column are not significantly different ( $Z$ test: $P=0.05$ ).

Citation from Hosokawa et al. (2004a).

Table 5. Shoot regeneration from LP-free SAMs of 'Piato' attached to cabbage root tips.

\begin{tabular}{cccccc}
\hline \hline $\begin{array}{c}\text { No. of repetitions of } \\
\text { LP-free SAM culture }\end{array}$ & $\begin{array}{c}\text { No. of LP-free } \\
\text { SAMs attached }\end{array}$ & $\begin{array}{c}\text { Percentage of viable } \\
\text { meristems }(\%)^{\mathrm{z}}\end{array}$ & $\begin{array}{c}\text { No. of plants with } \\
>3 \text { mm leaves }\end{array}$ & $\begin{array}{c}\text { No. of plants } \\
\text { subcultured }\end{array}$ & $\begin{array}{c}\text { Percentage of plants } \\
\text { with }>3 \text { mm leaves } \\
\text { after subculture }(\%)^{\mathrm{w}}\end{array}$ \\
\hline 1 & 180 & 65.6 & 0 & 115 & $79(68.7)$ \\
$2^{\mathrm{v}}$ & 30 & 53.3 & 0 & 14 & $6(42.9)$ \\
\hline
\end{tabular}

\footnotetext{
${ }^{z}$ Data were collected 10 days after attachment to root tips.

y Data were collected 30 days after attachment to root tips immediately before subculture on modified MS medium.

${ }^{x}$ No. of plants transferred to modified MS medium 30 days after attachment to root tips.

${ }^{\text {w }}$ Data were collected 30 days after subculture on modified MS medium.

(No. of plants with $3 \mathrm{~mm}$ leaves/Total no. of subcultured plants) $\times 100$

$\checkmark$ LP-SAMs of low-concentration CSVd-infected in vitro plants were used.

Citation from Hosokwa et al. (2004a).
} 
Table 6. CSVd-free plant production by LP-free SAM culture of 'Piato' attached to root tips of cabbage.

\begin{tabular}{cccc}
\hline \hline $\begin{array}{c}\text { No. of repetitions of LP-free } \\
\text { SAM culture }\end{array}$ & $\begin{array}{c}\text { No. of plants used for } \\
\text { CSVd detection }\end{array}$ & $\begin{array}{c}\text { No. of plants without CSVd signal } \\
\text { detected by RT-PCR (\%) }\end{array}$ & $\begin{array}{c}\text { No. of CSVd-free plants } \\
\text { detected by nested PCR (\%) }\end{array}$ \\
\hline 1 & 64 & $23(35.9) \mathrm{b}$ & $2(3.1) \mathrm{a}$ \\
$2^{\mathrm{w}}$ & 6 & $6(100) \mathrm{a}$ & $1(16.7) \mathrm{a}$ \\
\hline
\end{tabular}

${ }^{\mathrm{z}}$ (No. of plants without CSVd signal detected by RT-PCR/No. of plants used for CSVd detection) $\times 100$

y (No. of CSVd-free plants detected by nested PCR/No. of plants used for CSVd detection) $\times 100$

${ }^{w}$ LP-free SAMs of low-concentration CSVd-infected in vitro plants were used.

Values followed by the same letter in the column are not significantly different ( $\mathrm{Z}$ test: $P=0.05$ ).

Citation from Hosokawa et al. (2004a).

Table 7. CChMVd-free plant production by LP-free SAM culture of 'Piato' and 'Stettsuman' attached to cabbage root tips.

\begin{tabular}{lccc}
\hline \hline \multicolumn{1}{c}{ Cultivar } & $\begin{array}{c}\text { No. of plants used for } \\
\text { CChMVd detection }\end{array}$ & $\begin{array}{c}\text { No. of plants without CChMVd } \\
\text { signal detected by RT-PCR }(\%)^{z}\end{array}$ & $\begin{array}{c}\text { No. of CChMVd-free plants } \\
\text { detected by nested PCR (\%) }\end{array}$ \\
\hline Piato & 29 & $14(48.2)$ & $1(3.4)$ \\
Stettsuman & 6 & $2(33.3)$ & $2(33.3)$ \\
\hline
\end{tabular}

z (No. of plants without CChMVd signal detected by RT-PCR/No. of plants used for CChMVd detection) $\times 100$.

y (No. of CChMVd-free plants detected by nested PCR/No. of plants used for CChMVd detection) $\times 100$.

Citation from Hosokawa et al. (2005).

RT-PCR and nested PCR were conducted on 64 shoots (Table 6). CSVd was not detected in 23 shoots of all the tested plants by RT-PCR (Table 5). Moreover, CSVd was not detected in two plants by nested PCR (Table 6). Nested PCR showed that two plants were CSVd-free. Low-concentration CSVd-infected plants that did not generate a CSVd signal by RT-PCR but had a CSVd signal by nested PCR were propagated in vitro and using these in vitro plants, LP-free SAMs were cultured again on cabbage root tips. Six plants were regenerated from the second LP-free SAM culture (Table 5), and a CSVdfree plant could be obtained from LP-free SAMs of low-concentration CSVd-infected plants (Table 6). Therefore, low-concentration CSVd-infected plants for LP-free SAM culture may enhance the percentage of CSVd-free plant regeneration. CSVd was not detected in the free plants by nested PCR after 80-day cultivation.

When CSVd-free plants were grown in an experimental field, an interesting phenomenon was observed about a flowering trait of 'Piato' plants (Hosokawa et al., 2004c). In summer season in Japan, all CSVd-infected plants flowered autonomously even under long-day conditions, and the flowering of CSVd-infected plants regenerated from SAMs containing leaf primordia, was one month earlier than plants regenerated from CSVdinfected plants regenerated from LP-free SAMs. On the other hand, CSVd-free chrysanthemum plants regenerated from LP-free SAMs maintained their vegetative growth. When CSVd-free plants were inoculated again with CSVd by grafting them to CSVd-infected root stocks, they flowered autonomously again even under long-day conditions by night-break lighting. Results suggest that CSVd may control the qualitative development process, flowering; i.e., it can induce the autonomous flowering of chrysanthemum. Therefore, it is important to carefully observe plants from LP-free SAM, because when the parasite or symbiont, which controls some physiological or morphological reactions in plants, is eliminated, the phenotype of the plant may change.

\section{CChMVd as a model for Avsunviroidae}

Of the 29 plants of 'Piato' regenerated from LP-free SAMs attached to cabbage root tips, CChMVd was not detected in 14 plants by RT-PCR (Table 7). One regenerant from which the band corresponding to CChMVd was not detected by nested PCR (Table 7) was considered to be a CChMVd-free plant. The viroid was detected in 15 of the regenerated plants by RT-PCR; these plants are considered to be infected with CChMVd at high titer. Moreover, we obtained two CChMVd-free plants from 6 regenerated 'Stettsuman' plants (Table 7).

Three plants each of in vitro-grown CChMVd-free and -infected 'Stettsuman' plants regenerated from LPfree SAMs were transplanted to a plug tray. Disease symptoms such as chlorosis in newly expanded leaves were observed in one infected plant but not in the other two free plants.

\section{Candidate mechanisms for viroid elimination by LP-free SAM culture}

Viroid distribution near shoot tips

Combination methods of shoot-tip culture or shoottip grafting with temperature treatment or chemotherapy successfully produced viroid-free plants; however, in most cases, viroid detections were not performed by PCR-based methods. These free plants should be reevaluated so as to confirm whether viroids exist in these plants using more sensitive methods such as nested PCR or PCR hybridization. However, in this article, inferring the mechanism of viroid-free plant production by LPfree SAM culture is considered, including those previous reports. 
Momma and Takahashi (1983) supposed that HSVd does not exist in SAMs of hop plants as a result of culturing different sizes of shoot tip explants. Zhu et al. (2001) clarified by in situ hybridization that PSTVd belonging to Pospiviroidae moved through a tomato plant with photosynthate from the sink to the source. In this report, PSTVd could not be detected in the SAM of infected Nicotiana benthamiana and tomato plants; however, there is a possibility that the viroid titer in SAM is too low for detection using this method and even this experiment could not conclusively state that PSTVd did not exist in SAMs. Micro tissue-direct RTPCR (MT-direct RT-PCR) (Hosokawa et al., 2006b) is a simple method for detecting viroids in a very small tissue area. In this method, like the "colony direct PCR" method, plant sap on syringe needles placed in tissue is used for the RT template. When MT-direct RT-PCR was used for viroid detection, the detection sensitivities for CSVd and CChMVd were comparable to use for the template that was extracted and purified using Trizol (Invitrogen, USA). CSVd and CChMVd could be detected from most 'Piato' leaves infected by CSVd and CChMVd with a high titer. From low-titer plants, CSVd and CChMVd were detected only by nested PCR. When MT-direct RT products were used as templates for realtime PCR, the CSVd titer in chrysanthemum plants decreased in the upper portion, and CSVd was not detected in the very tips of SAMs by real-time PCR (Hosokawa et al., 2006a).

In contrast, Avocado sunblotch viroid (ASBVd), belonging to Avsunviroidae, cannot be eliminated by shoot tip grafting, for which high-titer ASBVd-infected avocado meristems containing 2 or 3 leaf primordia were used (Suarez et al., 2005). Rodio et al. (2007) revealed that, using in situ hybridization, Peach latent mosaic viroid (PLMVd), belonging to Avsunviroidae, could invade SAM except at the very tip of peach plants. A minus strand of PLMVd is also observed in SAMs, which indicates that PLMVd RNA not only exists in SAMs but also proliferates there. They also ascertained that ASBVd can invade SAMs of avocado plants. MT-direct RT-PCR using chrysanthemum 'Piato' plants also suggested that CChMVd can invade SAMs and the titer in a SAM is almost the same as in other organs, such as leaves, stems, and shoot tips (Hosokawa et al., unpublished data). It is reported that there is a barrier for selective RNA invasion to SAMs (Foster et al., 2002). It is supposed that CChMVd RNA can pass through this barrier, which complicates the reason why LP-SAM culture can eliminate CChMVd from chrysanthemum 'Piato' plants.

However, it is too early to conclude that Avsunviroidae has a special ability to invade SAMs and the ability is limited in Pospiviroidae. In SAMs of the chrysanthemum 'C-7' cultivar, which we bred as a CSVd-resistant plant, no CSVd signal was detected even below the SAM and the leaf primordial, and in contrast to this plant, in the
SAM of 'A-4' cultivar, which was screened as a CSVdsensitive plant, the signal of CSVd was detected in SAMs without the corpus region (Omori et al., unpublished data). Hence, the CSVd-infecting area near SAMs is dependent not only on viroid species but also on cultivars whose effect will be caused by the RNA invasion barrier, lack of RNA proliferation or RNA breakdown ability.

Post-transcriptional gene silencing mechanism in SAMs

One of the roles of post-transcriptional gene silencing (PTGS) for a plant is the inhibition of virus proliferation, as explained by many reviews such as by Vaucheret and Fagard (2001). Several reports observed small interference RNAs (siRNAs) from viroid-infected plants, which are regarded as markers for PTGS (Itaya et al., 2001; Martínez de Alba et al., 2002; Papaefthimiou et al., 2001). Moreover, a relationship between RNA silencing and recovery from viroid pathogenicity was observed (Markarian et al., 2004; Sano and Matsuura, 2003).

PTGS on cucumber mosaic virus (CMV) RNA was observed in virus-infected tobacco SAMs (Mochizuki and Ohki, 2004). Inoculated CMV successfully invaded SAMs and accumulated, the virus titer then decreased, and finally could not be detected by in situ hybridization. SiRNA, which is a product of CMV breakdown, was detected in relation to CMV accumulation in SAMs. This result clearly explains why CMV cannot be detected in SAMs, since it is not the invasion of CMV into SAMs but RNA breakdown in SAMs. Matoušek et al. (2001) found HLVd-RNA cleaved products which were longer than si and microRNAs when thermotherapy was used to treat infected plants, and they suggested that there are other mechanisms concerning RNA breakdown about viroids.

Rodio et al. (2007) detected plus and minus strands of PLMVd in PLMVd-infected SAMs; however, at the very tips of SAMs, no signal was observed. Moreover, in CSVd, plus and minus strands were not observed in the corpus region of SAMs even in the very sensitive cultivar 'A-4', which indicates that SAMs have some special abilities for inhibiting invasion or proliferation of the viroid. Unfortunately it cannot be explained why CChMVd was eliminated by LP-free SAM culture because CChMVd can proliferate and accumulate in SAMs. Some unknown effects of the isolation of very small meristems from the plants may explain viroid disappearance.

\section{Future Prospects}

LP-free SAM culture is a suitable method for the elimination of viroids that cannot be eliminated by conventional methods, such as shoot-tip culture or shoottip grafting. In addition to viroids, tobacco streak virus (TSV), one of the most difficult pathogens to eliminate could be eliminated from a high titer-infected dahlia plant using LP-free SAM culture (Hosokawa et al., unpublished data). 
As pointed out by Diener (1995), during the last 150 to 200 years, vegetative cuttings of horticultural crops have been moved long distances and this crop movement triggered the occurrence of viroid problem. Bar-Joseph (2003) mentioned that the new era of Hort-Technology has not only enabled major horticultural improvements but has also sporadically caused the re-emergence of viroid disease problems at the meeting places between infected tolerant cultivars and the introduction of sensitive genotypes. Recently, it was reported by Verhoeven et al. (2008) that vegetatively propagated ornamental plants infected with PSTVd are supposed to act as sources of PSTVd in tomato plants. In addition, sometimes two different viroid species meet in a plant to generate a new chimeric viroid (Sano and Ishiguro, 1998), and it is daunting to consider new viroid species generation.

Infrastructures, such as quarantine facilities, and new techniques for viroid detection and elimination are needed to protect important crops from viroid infection. For field crop production, epidemiological research is important. From a horticultural viewpoint, it is important to establish new techniques for detecting and eliminating viroid, and a propagation system for viroid-free plants. In addition, viroid-resistant cultivars should be bred to keep the viroid titer low in planta and to avoid scattering viroid molecules to other crops.

For important horticultural crops, phytosanitary programs developed by individual countries, such as in the EU or North America, sometimes include rules for viroids (Barba et al., 2003a). In quarantine facilities, laborious and long-term inspections are conducted before issuing phytosanitary certification. In the future, to protect crops from newly-arrived pathogens, the regulatory framework for importing and exporting plants, seeds or cuttings should be improved. Using in vitro plants with certified by private companies is one of ways for the safety trade. Thus, the production of plants free from difficult-to-eliminate pathogens, such as viroids, is an important horticultural role not only for the restoration of plants to their original state, including reinvigoration, but also to supply high-quality plants for trade.

\section{Literature Cited}

Adams, A., D. J. Barbara, A. Morton and P. Darby. 1996. The experimental transmission of hop latent viroid and its elimination by low temperature treatment and meristem culture. Ann. Appl. Biol. 128: 37-44.

Ball, E. 1948. Differentiation in the primary shoots of Lupinus albus L. and of Tropaeolum majus L. Symp. Soc. Exp. Biol. 2: 246-262.

Barba, M., S. Cupidi, S. Loreti, F. Faggioli and L. Martino. 1995. In vitro micrografting: a technique to eliminate peach latent mosaic viroid from peach. Acta Hort. 386: 531-535.

Barba, M., Gumpf, D. J. and A. Hadidi. 2003a. Quarantine of imported germplasm. p. 303-311. In: A. Hadidi, R. Flores, J. W. Randles and J. S. Semanick (eds.). Viroids. CSIRO,
Collingwood.

Barba, M., E. Ragozzino and L. Navvaro. 2003b. Viroid elimination by thermotherapy and tissue culture. p. 318-323. In: A. Hadidi, R. Flores, J. W. Randles and J. S. Semanick (eds.). Viroids. CSIRO, Collingwood.

Bar-Joseph, M. 2003. Natural history of viroids-Horticultural aspects. p. 246-251. In: A. Hadidi, R. Flores, J. W. Randles and J. S. Semanick (eds.). Viroids. CSIRO, Collingwood.

Brierley, P. and F. F. Smith. 1949. Chrysanthemum stunt. Phytopathol. 39: 501-509.

Diener, T. O. 1991. Subviral pathogens of plants: viroids and viroidlike satellite RNAs. FASEB J. 5: 2808-2813.

Diener, T. O. 1995. Origin and evolution of viroids and viroidlike satellite RNAs. Virus Genes 11: 119-131.

Dimock, A. W. 1947. Chrysanthemum stunt. New York State Flower Growers Bull. 26: 2.

Ding, B. and A. Itaya. 2007. Viroid: A useful model for studying the basic principles of infection and RNA biology. Mol. PlantMicrobe Interact. 20: 7-20.

Ding, B., A. Itaya and X. Zhong. 2005. Viroid trafficking: a small RNA makes a big move. Curr. Opin. Plant Biol. 8: 606-612.

Flores, R., C. Hernández, A. E. Martínez de Alba, J-A. Daròs and F. Di Serio. 2005. Viroids and Viroid-Host Interactions. Annu. Rev. Plant Pathol. 47: 117-139.

Flores, R., J-A. Daròs and C. Hernández. 2000a. The Avsunviroidae family: viroids containing hammerhead ribozymes. Adv. Virus Res. 55: 271-323.

Flores, R., J-A. Daròs and J. A. Navarro. 2003. Replication. p. 55-60. In: A. Hadidi, R. Flores, J. W. Randles and J. S. Semancik (eds.). Viroids. CSIRO, Collingwood.

Flores, R., J. W. Randles, M. Bar-Josef and T. O. Diener. 2000b. Subviral agents: Viroids. p. 1009-1024. In: M. H. V. van Regenmortel, C. R. Pringle and R. B. Wickner (eds.). Virus Taxonomy, Seventh Reports of the International Committee on Taxonomy of Viruses. Academic Press, San Diego.

Foster, T. M., T. J. Lough, S. J. Enerson, R. H. Lee, J. L. Bowman, R. L. S. Forester and W. J. Lucas. 2002. A survelillance system regulates selective entry of RNA into the shoot apex. Plant Cell 14: 1497-1508.

Francki, R. I. B., M. Zaitlin and P. Palukaitis. 1986. In vitro encapsidation of potato spindle tuber viroid by velvet tobacco mottle virus particles. Virology 155: 469-473.

Hirata, Y., Y. Yamashita, T. Sano and H. Fukui. 2002. Detection of CSVd-RNA by RT coupled loop-mediated isothermal amplification. J. Japan. Soc. Hort. Sci. 71 (Suppl. 2): 261 (In Japanese).

Hollings, M. and O. M. Stone. 1973. Attempts to eliminate chrysanthemum stunt from chrysanthemum by meristem-tip culture after heat-treatment. Ann. Appl. Biol. 73: 333-348.

Horst, R. K. and D. Cohen. 1980. Amantadine supplement tissue culture medium: a method for obtaining chrysanthemum free of chrysanthemum stunt viroid. Acta Hort. 110: 311-315.

Horst, R. K. and P. Langhans. 1977. Effect of chrysanthemum stunt, chlorotic mottle, aspermy and mosaic on flowering and rooting of chrysanthemums. Phytopathol. 67: 9-14.

Hosokawa, M., A. Otake, K. Ohishi, E. Ueda, T. Hayashi and S. Yazawa. 2004a. Elimination of chrysanthemum stunt viroid from an infected chrysanthemum cultivar by shoot regeneration from a leaf primordium-free shoot apical meristem dome attached to a root tip. Plant Cell Rep. 22: 859-863.

Hosokawa, M., A. Otake, Y. Sugawara, T. Hayashi and S. Yazawa. 2004b. Rescue of shoot apical meristems of chrysanthemum by culturing on root tips. Plant Cell Rep. 22: 443-448.

Hosokawa, M., E. Ueda, K. Ohishi, A. Otake and S. Yazawa. 
2004c. Chrysanthemum stunt viroid disturbs the photoperiodic response for flowering of chrysanthemum plants. Planta 220: 64-70.

Hosokawa, M., S. Katsumura, T. Hayashi and S. Yazawa. 2004d. Plant regeneration of stable parthenocarpic tomato cultivars from shoot apical meristems attached to root tips. Jpn. J. Taste Smell Res. 11: 61-68 (In Japanese).

Hosokawa, M., T. Kawabe and S. Yazawa. 2006a. Detection of two viroids in shoot apical meristems of chrysanthemum. J. Japan. Soc. Hort. Sci. 75 (Suppl. 1): 408 (In Japanese).

Hosokawa, M., Y. Matsushita, H. Uchida and S. Yazawa. 2006b. Direct RT-PCR method for detecting two chrysanthemum viroids using minimal amounts of plant tissue. J. Virol. Meth. 131: $28-33$.

Hosokawa, M., Y. Matsushita, K. Ohishi and S. Yazawa. 2005. Elimination of chrysanthemum chlorotic mottle viroid (CChMVd) recently detected in Japan by leaf-primordia free shoot apical meristem culture from infected cultivars. J. Japan. Soc. Hort. Sci. 74: 386-391.

Howell, W. E., J. Burgess, G. I. Mink, L. J. Skrzeczkowski and Y. P. Zang. 1998. Elimination of apple fruit and bark deforming agents by heat therapy. Acta Hort. 472: 641-646.

Itaya, A., A. Folimonov, Y. Matsuda, R. S. Nelson and B. Ding. 2001. Potato spindle tuber viroid as inducer of RNA silencing in infected tomato. Mol. Plant-Microbe Interact. 14: 13321334.

Markarian, N., H. W. Li, S. W. Ding and J. S. Semancik. 2004. RNA silencing as related to viroid induced symptom expression. Arch. Virol. 149: 397-406.

Martínez de Alba, A. E., R. Flores and C. Hernández. 2002. Two chloroplastic viroids induce the accumulation of small RNAs associated with posttranscriptional gene silencing. J. Virol. 76: 13094-13096.

Matoušek, J., J. Patzak, L Orctová, J. Schubert, L. Vrba, G. Steger and D. Riesner. 2001. The variability of hop latent viroid as induced upon heat treatment. Virology 287: 349-358.

Mink, G. I. 1993. Pollen- and seed-transmitted virus and viroids. Annu. Rev. Phytopathol. 31: 375-402.

Mochizuki, T. and S. T. Ohki. 2004. Shoot meristem tissue of tobacco inoculated with Cucumber mosaic virus is infected with the virus and subsequently recovers from infection by RNA silencing. J. Gen. Plant Pathol. 70: 363-366.

Momma, T. and T. Takahashi. 1983. Cytopathology of shoot apical meristem of hop plants infected with hop stunt viroid. Phytopath. Z. 106: 272-280.

Murashige, T., W. P. Bitters, E. M. Rangan, E. M. Nauer, C. N. Roistacher and P. B. Holliday. 1972. A technique of shoot apex grafting and its utilization toward recovering virus-free Citrus clones. HortScience 7: 118-119.

Notomi, T., H. Okayama, H. Masubuchi, T. Yonekawa, K. Watanabe, N. Amino and T. Hase. 2000. Loop-mediated isothermal amplification of DNA. Nucl. Acids Res. 28: E63.

Ohishi, K., Y. Okumura and K. Morioka. 2001. Transmission of chrysanthemum stunt viroid through seed of chrysanthemum.
J. Japan. Soc. Hort. Sci. 70 (Suppl. 2): 192 (In Japanese).

Papaefthimiou, I., A. J. Hamilton, M. A. Denti, D. C. Baulcombe, M. Tsagris and M. Tabler. 2001. Replicating potato spindle tuber viroid is accompanied by short RNA fragments that are characteristic of post-transcriptional gene silencing. Nucl. Acids Res. 29: 2395-2400.

Randles, J. W. 2003. Economic impact of viroid diseases. p. 3 11. In: A. Hadidi, R. Flores, J. W. Randles and J. S. Semanick (eds.). Viroids. CSIRO, Collingwood.

Rodio, M., S. Delgado, A. De Stradis, M. Gómez, R. Flores and F. Di Serio. 2007. A viroid RNA with a specific structural motif inhibits chloroplast development. Plant Cell 19: 3610 3626.

Salazar, L. F., M. Querci, I. Bartolini and V. Lazarte. 1995. Aphid transmission of potato spindle tuber viroid assisted by potato leafroll virus. Fitopatologia 30: 56-58.

Sano, T. and A. Ishiguro. 1998. Viability and pathogenicity of inter-subgroup viroid chimeras suggest possible involvement of the terminal right region in replication. Virology 240: 238 244.

Sano, T. and Y. Matsuura. 2003. Accumulation of short interfering RNAs characteristic of RNA silencing precedes recovery of tomato plants from severe symptoms of Potato spindle tuber viroid infection. J. Gen. Plant Pathol. 70: 50-53.

Semanic, J. S., D. Tsuruda, L. Zaner, J. L. M. C. Green and J. G. Weathers. 1976. Exocortis disease: Subcellular distribution of pathogenic (viroid) RNA. Virology 69: 669-676.

Steger, G. and D. Riesner. 2003. Molecular characteristics. p. 1529. In: A. Hadidi, R. Flores, J. W. Randles and J. S. Semanick (eds.). Viroids. CSIRO, Collingwood.

Suarez, I. E., A. S. Raymond, D. N. Kuhn and R. E. Litz. 2005. Micrografting of ASBVd-infected avocado (Persea americana) plants. Plant Cell Tiss. Org. Cult. 80: 179-185.

Sugiura, H. and K. Hanada. 1998. Chrysanthemum stunt viroid, a disease of large-flowered chrysanthemum in Niigata Prefecture. J. Japan. Soc. Hort. Sci. 67: 432-438.

Sussex, I. M. 1952. Regeneration of the potato shoot apex. Nature 170: 755-757.

Tabler, M. and M. Tsagris. 2004. Viroids: petite RNA pathogens with distinguished talents. Trends Plant Sci. 9: 339-348.

Vaucheret, H. and M. Fagard. 2001. Transcriptional gene silencing in plants: Targets, inducers and regulators. Trends Genet. 17: 29-35.

Verhoeven, J. Th. J., C. Jansen, M. Botermans and A. Roenhorst. 2008. Evidence that vegetatively-propagated solanaceous plant species act as sources of Potato spindle tuber viroid infection for tomato. Proceedings of International Symposium Viroid and Viroid-like RNAs 2008: 27.

Yamamoto, H. and T. Sano. 2005. Occurrence of Chrysanthemum chlorotic mottle viroid in Japan. J. Gen. Plant Pathol. 71: 156-157.

Zhu, Y., L. Green, Y-M. Woo, R. Owens and B. Ding. 2001. Cellular basis of potato spindle tuber viroid systemic movement. Virology 279: 69-77. 